\title{
Double digital watermarking algorithm based on SVD - DFT technologies
}

\author{
ZHENG Shao-hua ${ }^{1}$, XU Cai Yun ${ }^{2}$, PEI Xiao-gen ${ }^{1,3}$ \\ (1. Communication command department of the Armored Force Institute, \\ bengbu Anhui, China; 2. Simulation training center of the Armored Force \\ Institute, bengbu Anhui, China; 3. School of Information Science and \\ Engineeri ng, Shandong University, Jinan 250100)
}

\begin{abstract}
In view of the current most based on Fourier transform domain method, its resist geometric attacks performance is very good, the embedded information is also big, but there are shearing attack ability is bad problems, and put forward a kind of based on singular value decomposition and discrete Fourier transform with the combination of double watermarking algorithm is proposed. In this scheme, first to do image singular value decomposition, and then out of decomposition of matrix DFT spectrum amplitude image watermark embedding, namely the row of molecules, to each image block DFT transform, relative to a the predefined threshold selection important coefficient, in its corresponding to the extent of the component watermark information, the weight of the original position and embedded watermark extraction preserved as a key, you will get the image with the watermark. The experimental results show that this method can achieve good image visual effect, to shear, noise, and rotated against all has good robustness.
\end{abstract}

Keywords: SVD-DFT algorithm; Dual digital watermarking; algorithm

\section{Introduction}

Along with the digital watermarking digital products in the extensive use of humanity in its recognition of the importance of increasing also, that prompted many experts and scholars with digital watermarking ongoing research, make the digital watermarking technology development level there is a qualitative leap: that is a heavy watermark by the original design development up to now the double watermark design even multiple watermarks design scheme. Such as Peter h. w. Wong $^{[1]}$, this paper proposes a practical for the image of the new multiple blind watermarking technology, this algorithm for different purpose Suggestions will watermarking embedding digital image, the watermark is designed for decoding or detection without the original image. The experimental results show that, using the proposed watermarking embedding technology can provide good image quality, and in different degree of JPEG compression, low pass filtering, noise pollution, print and scan the attacks have strong robustness. With some experts and scholars on 
double digital watermarking in image processing applications have advanced, in the DCT-SVD and their combining research aspects in succession some digital watermarking paper ${ }^{[2-6]}$ published, but so far, it is at present in their combining SVD and DFT of dual digital watermarking research, there is no such published research articles. This paper will put forward their combining digital watermarking scheme of double, SVD embedded in the image watermark image, and on the basis of watermark image again to contain DFT validation watermarking embedding, and the host image watermark embedding double, with the theoretical and experimental results verify that the double watermarking scheme design of feasibility.

\section{SVD and DFT combination of double watermarking scheme}

Double watermark: just as its name implies, is to have two watermarks. In fact it can be understood as to have at least two watermark, here also contains more than two of the watermark the implication, because some algorithm is likely watermark nested the watermark, clearly two watermarking algorithm loading together, but does not say double watermark (only as a kind of new algorithm-or a heavy watermark $^{[7]}$ ) and so on. In view of the SVD and DFT in the combination of digital watermark in the blank of double literature, specially put forward the design, this also is the core content of this paper. Generally speaking, the watermarking system is always by watermarking embedding watermarking extraction and two main components (special requirements for the verification of except), so this paper in the overall arrangement for and by the way on design, just in the content on the rich and modification, concrete plan is as follows:

\subsection{Watermarking embedding}

Considering the SVD algorithm of geometric distortion in the image has strong robustness and DFT algorithm has very good shearing ability, so choose to use the two algorithm embedded watermark. In the watermark embedding method on the overall design of the two framework implementation: the first using SVD watermark embedding algorithm, acquiring a carrier images, call one of; To get the carrier images in use the DFT secondary watermark embedding algorithm, which can get double the carrier image with the watermark, this in two. In the carrier image watermarking embedding before, here to definition:

Define 1. for $N \times N$ matrix $A$, if there is $N$ scalar $\lambda_{i}(i=1,2, \cdots, N)$ meet:

$$
\left|A-\lambda_{i} I\right|=0
$$

It can be said that the group $\lambda_{i}$ for the characteristic value of the matrix $A$.

Definition 2. if there is such $N \times 1$ vector $V_{i}$, are: 


$$
A V_{i}=\lambda_{i} V_{i}
$$

Here the term $V_{i}$ for and $A$ characteristic value $\lambda_{i}$ and the corresponding feature vector. $A$ total of $N$ feature vector.

Definition 3. Singular Value Decomposition (SVD: Singular Value Decomposition) is the inherent characteristics of the matrix, in the role of the digital image processing is very important. This hypothesis matrix $A \in R^{m \times n}$, $\operatorname{rank}(A)=r, r \leq n$, So the singular value decomposition of matrix $A$ are defined as follows:

$$
A=U D V^{T}=\left[u_{1}, u_{2}, \cdots, u_{m}\right]\left[\begin{array}{l}
\sigma_{1} 0 \cdots 0 \\
\vdots \\
00 \cdots \sigma_{r}
\end{array}\right]_{m \times n}\left[v_{1}, v_{2}, \cdots, v_{n}\right]^{T}=\sum_{j=1}^{r} \sigma_{j} u_{j} v_{j}
$$

Among them, $U=\left[u_{1}, \cdots u_{m}\right] \in R^{m \times m}$ and $V=\left[v_{1}, \cdots v_{n}\right] \in R^{n \times n}$ are orthogonal matrixs. The column for $u_{i}$ and $v_{i}$ respectively. Here $\mathrm{U}, \mathrm{V}$ respectively is matrix A right and left singular matrix; $\mathrm{D}$ is diagonal matrix; $\sigma_{i}(i=1, \cdots, r)$ known as the matrix $A$ of the singular value, Here is $A A^{T}$ and $A^{T} A$ the characteristic value $\lambda_{i}$ of positive or square root and satisfied $\sigma_{1} \geq \sigma_{2} \geq \cdots \geq \sigma_{r}>\sigma_{r+1}=\cdots=\sigma_{m}=0$. Due to the matrix singular value has the very good stability, and that is when the matrix A slight vibration, the singular value for the change, change the size also not greater than 2-the vibration matrix norm. In a certain extent decomposition of matrix $S V D$ can be used in image compression. For image the decomposed singular value matrix $\Sigma$, some of the smaller diagonal yuan for zero, and then to $S V D$ inverse transform can complete image lossy compression. The basic principle of the $S V D$ algorithm is: first to digital image image matrix, then the image of a matrix $A_{m \times n}$ singular value decomposition, will get two orthogonal matrix $U$ and $V$, and a diagonal matrix $S$, and the watermark is embedded again to this diagonal matrix $S$, the watermark $W \in R^{n \times n}$ by adding to the matrix $S$, then the new produce singular value decomposition of matrix $S+\alpha W$, still can get $U_{1}$ and $S_{1}$ and $V_{1}\left(S+\alpha W=U_{1} S_{1} V_{1}^{T}\right)$, 
one constant $\alpha>0$ is the superposition of watermark strength, and then will matrix $U$ and $S_{1}$ and $V^{T}$ multiplication, can get treatment of common image $\hat{A}$. Here, on behalf of the matrix $A$ and $W$ the original image matrix and digital watermark. SVD algorithm and DFT algorithm through the following of the eight steps can get double the watermark with complex carrier images $\hat{A}$, specific watermarking embedding procedure is as follows:

Step1: Firstly, the original image of the matrix, and then the image matrix $A$ of the singular value decomposition:

$$
A=U S V^{T}
$$

Step2: Read the watermark image $W$, will the superposition to image the diagonal matrix $S$ decomposition of matrix out the new matrix $S^{\prime}$ :

$$
S^{\prime}=S+\alpha W
$$

Step3: In the digital watermarking embedding, then to produce new singular value decomposition of matrix $S^{\prime}$ :

$$
S^{\prime}=U_{1} S_{1} V_{1}^{T}
$$

Step4 : Then will the new matrix decomposition of orthogonal matrix multiplication $S_{1}$ and $V^{T}$ and $U$, with the watermark can get image:

$$
\hat{A}=U S_{1} V^{T}
$$

In order to further improve the safety of image transmission robustness and, in the need to contain the image watermark algorithm using two times, DFT watermark embedding. At present the discrete Fourier transform research direction to basically have two kinds: one is based on the domain of DFT translations, scales and rotation of the geometry of the constant, will the carrier image Fouier-Millin transformation to realize the embedded watermark to resist geometric attacks. But this kind of algorithm for use among them interpolation approximate computation, watermarking embedding will cause the distortion of the larger after, and complex algorithm, realize up the difficulty is large. Another kind is using Fourier domain of the spectrum of information to embed watermark, the study found that the watermark should be inserted in the important component to get good robustness, the embedded method mainly have the spectrum amplitude, phase spectrum, phase and amplitude spectrum on some, this paper used the DFT spectrum amplitude watermarking algorithm is proposed. Its principle is: the first original image of stroke molecules, to each image block DFT transform, relative to a the predefined threshold selection important coefficient, in its corresponding to the extent of the component watermark 
information, the weight of the original position and embedded watermark extraction preserved as a key, you will get the image with the watermark. DFT spectrum amplitude watermarking embedding algorithm flow diagram see figure 1:

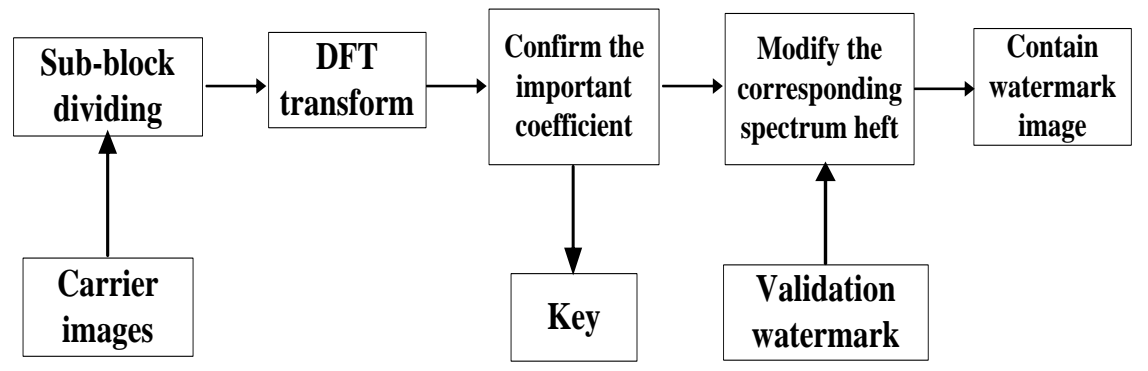

Figure 1 DFT spectrum amplitude embedding algorithm flow diagram

Because in the watermark image DFT frequency domain regional watermark, easy to be human eye notice, but would also reduce the watermark image quality; As in areas of high frequency watermark, it's easy to be JPEG compression, low pass filtering common image processing is marred by operation, its robustness is poorer. So, here to choose in the image of the son of intermediate frequency area DFT domain watermark signal. DFT spectrum amplitude watermarking algorithm is concrete is:

Step5 : The carrier image sub-block dividing: first the carrier image $8 \times 8$ into of no cover image of the son.

$$
\begin{gathered}
f(m, n)=\bigcup_{K=0}^{K-1} f_{k}\left(m^{\prime}, n^{\prime}\right) \\
0 \leq m^{\prime}, n^{\prime}<8
\end{gathered}
$$

Step6: Again to each image one sub-block DFT transformation:

$$
\begin{gathered}
F_{k}\left(u^{\prime}, v^{\prime}\right)=D F T\left\{f_{k}\left(m^{\prime}, n^{\prime}\right), 0 \leq m^{\prime}, n^{\prime}<8\right\} \\
0 \leq u^{\prime}, v^{\prime}<8
\end{gathered}
$$

Step7: And then determine the important coefficient of the spectrum. If coefficient meet:

$$
\left|F_{k}(r, c)\right|^{2} /(1 / 64) \sum_{u^{\prime}=0}^{7} \sum_{v^{\prime}=0}^{7}\left|F_{k}\left(u^{\prime}, v^{\prime}\right)\right|^{2}>t
$$

The $F_{k}(r, c)$ relative to a specified threshold $t$ in advance is the important coefficient, this is equivalent to the coefficient of frequency domain to choose, using a low frequency coefficients corresponding to the watermark weight to improve the 
robustness of the algorithms. In addition, it is worth noting that, in the process of selecting the important coefficient of the generation of extraction of watermark key, that is used to embed watermark information of the position and the corresponding spectrum component.

Step8: Then again of the spectrum component change them accordingly. Because the DFT spectrum amplitude domain with symmetry, in order to maintain this symmetry after embedding watermarking unchanged, and also to ensure that recovery image pixels for real number, the embedded watermark symmetrical, namely:

$$
\begin{aligned}
& \text { Amplitude }\left(F_{k}^{\prime}(r, c)\right) \leftarrow \text { Amplitude }\left(F_{k}(r, c)\right)+\alpha m_{i} \\
& \text { Amplitude }\left(F_{k}^{\prime}(7-r, 7-c)\right) \leftarrow \operatorname{Amplitude}\left(F_{k}(7-r, 7-c)\right)+\alpha m_{i}
\end{aligned}
$$

In the type Amplitude $(\bullet)$ to the corresponding amount of amplitude spectrum, $m_{i}$ for watermarking information and $\alpha$ for constant, said the amplitude of the watermarking embedding strength. In the watermark information select validation watermark image pixels for $40 \times 40$ here, if the carrier image pixels for $384 \times 384$, so they can get 1600 pixels $8 \times 8$ factor matrix, and factor matrix is part of the top left corner of the low frequency components, lower the high coefficients is part, finally obtained the carrying of the watermark image complex double carrier $A$.

\subsection{Watermarking extraction.}

For double watermarking scheme for, the watermarking algorithm is still the imbedding algorithm is the inverse of the process. The algomithm of the watermark extraction process is: first to answer the carrier image $A$ watermark extraction SVD algorithm, if in the watermark detection process, have given matrix $U_{1}$ and $S$ and $V_{1}$, and the possible damage to the watermark image $A^{*}$, it can be extracted by inverse process may have distortion of the watermark $W^{*}$, watermarking extraction procedure is as follows:

Step1: For complex carrier images, and the watermark is damaged the unknown, and that may damage the watermark here (to plan for the worst), but also want to watermark image $A^{*}$ of the singular value decomposition, in order to verify the integrity of the:

$$
A^{*}=U^{*} S_{1}^{*} V^{* T}
$$

Step2: Then calculation middle matrix:

$$
D^{*}=U_{1} S_{1}^{*} V_{1}^{T}
$$


Step3: And get a need to digital watermarking images:

$$
W^{*}=\frac{1}{\alpha}\left(D^{*}-S\right)
$$

Here is $\alpha$ to adjust the superposition of watermark strength, in the experimental process SVD found that its value is not the bigger the better, such as too big can make contain the concealment of the watermark image become worse, see figure 2 shows; After many SVD after the experiment found, adjust the superposition of values of the strength of the watermark $\alpha$ is a moderate range, general $\alpha=0-0.18$ with advisable.

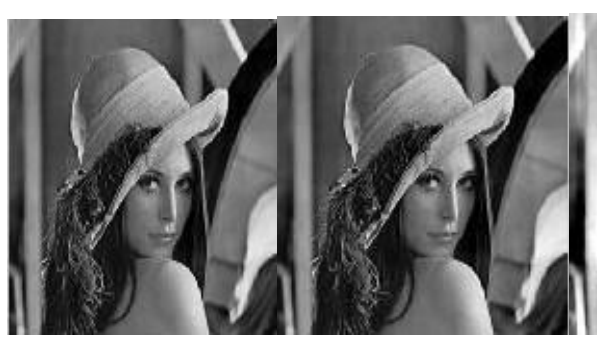

(a) (b)

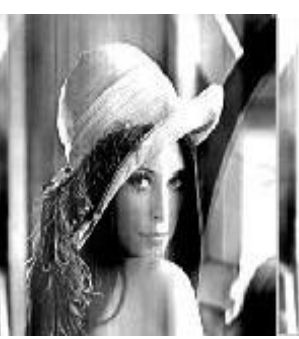

(c)

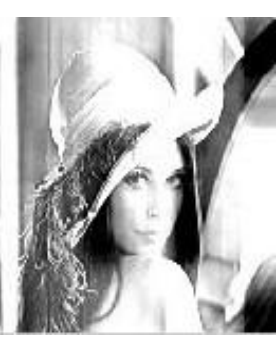

(d) ( (a) $\alpha=0.18$;

(b) $\alpha=0.2$; (c) $\alpha=1$;

(d) $\alpha=1.5$ )

Figure $2 \alpha$ different selection on the influence of the carrier images

In order to verify above through the SVD watermarking extraction method of reliability and safety, it is necessary to verify, here to take the verification method and different (past is mostly for the extraction of the watermark verification), the complex carrier images to continue to extract of the watermark security methods to verification, extraction process using IDFT method, the concrete validation watermark extraction procedure is as follows:

Step4: To answer the carrier image watermark extraction DFT the process, is the inverse of the embedded in its process, a process called watermarking extraction in the first time after the complex carrier images to each child block inverse discrete Fourier transform (IDFT), and embedding algorithm is proposed according to the same threshold $t$ confirm the important coefficient, using embedded preserved when extracted key embedded watermark, and ultimately the validation watermark image.

$$
\begin{gathered}
\left\{f_{k}^{\prime}\left(m^{\prime}, n^{\prime}\right), 0 \leq m^{\prime}, n^{\prime}<8\right\}=\operatorname{IDFT}\left\{f_{k}\left(m^{\prime}, n^{\prime}\right), 0 \leq m^{\prime}, n^{\prime}<8\right\} \\
f^{\prime}(m, n)=\bigcup_{k=0}^{k-1} f_{k}^{\prime}\left(m^{\prime}, n^{\prime}\right)
\end{gathered}
$$


Through to the watermarking extraction and verified with the original image watermark compared respectively, which can be confirmed that the scheme of security and robustness.

\section{The simulation experiment}

The simulation experiment selection of watermark image for 384 x 384 binary images, host image for $384 \times 384$ Lena of gray image, the simulation platform software use matlab7.0. Embedded watermark image quality objective appraisal USES the PSNR to measure, and the watermark is an objective evaluation of the testing results of the normalized correlation coefficient $\mathrm{NC}$ to measure. In order to validate the robustness of the algorithms, this paper take the following experiment:

\subsection{Shear against the experiment.}

First carrier watermark image shear experiments, figure 3 (a) said $30 \%$ of the shear area containing watermark image, then the PSNR $=6.8600 \mathrm{~dB}$. Through the figure 3 (b) can see despite the watermark extraction have slash, but the watermark extraction or very clear, the $\mathrm{NC}=0.9979$. Through the table 1 of the specific experiment data can be found when the shear area as high as $50 \%$, still can detect watermarking existence.

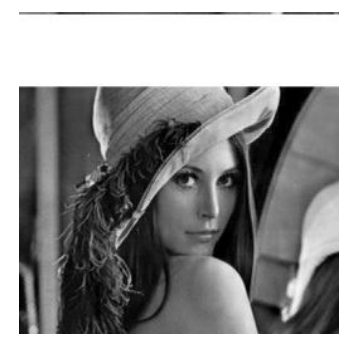

(a)

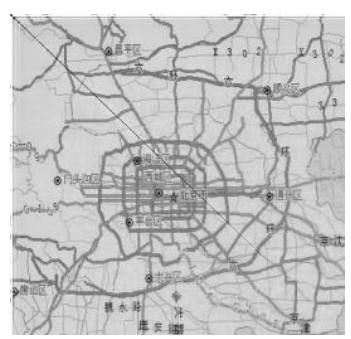

(b)

Figure $330 \%$ of the complex shear after carrier images and extraction of the original image

Second verification watermark image shear experiments, the pixels for $40 \times 40$ of the anti-counterfeiting mark text seal Stamper. BMP binary image as a validation watermark, and figure 4 (a) said $30 \%$ of the shear area containing watermark image, then the PSNR $=6.8600 \mathrm{~dB}$. Through the figure $4(\mathrm{~b})$ can see despite the watermark extraction, there are many shading points, but the watermark extraction is still clear, the $\mathrm{NC}=0.9184$. Through the table 2 of the specific experimental data can be found when the shear area as high as $50 \%$, still can detect watermarking existence. 


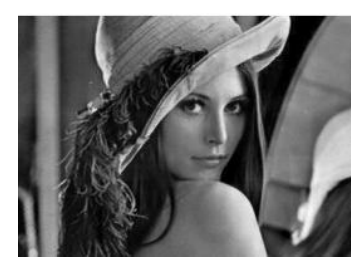

(a)

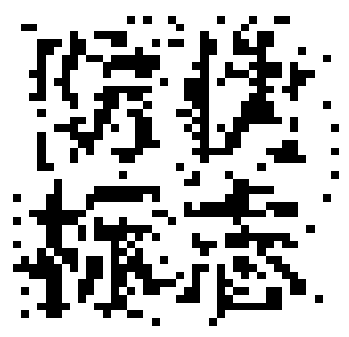

(b)

Figure $430 \%$ of the complex shear after carrier images and extraction of the original image TABLE I Contain the watermark grayscale image shear experimental data (carrier watermark image shear experiment data)

\begin{tabular}{c|c|c|c|c|c}
\hline $\begin{array}{r}\text { Shear } \\
\text { rate } \\
\text { content }\end{array}$ & $10 \%$ & $20 \%$ & $30 \%$ & $40 \%$ & $50 \%$ \\
\hline PSNR & 14.8306 & 9.1246 & 6.8600 & 6.0525 & 3.7928 \\
\hline NC & 0.9986 & 0.9984 & 0.9979 & 0.9972 & 0.9968 \\
\hline
\end{tabular}

TABLE II Contain the watermark grayscale image shear experimental data (verification watermark image shear experimental data)

\begin{tabular}{c|c|c|c|c|c}
$\begin{array}{r}\text { Shear } \\
\text { rate } \\
\text { content }\end{array}$ & $10 \%$ & $20 \%$ & $30 \%$ & $40 \%$ & $50 \%$ \\
\hline PSNR & 14.8306 & 9.1246 & 6.8600 & 6.0525 & 3.7928 \\
\hline NC & 0.9379 & 0.9197 & 0.9184 & 0.9169 & 0.8840 \\
\hline
\end{tabular}

In comparison of the various data, can see, SVD algorithm is better than the DFT algorithm, the reason is, on the whole, SVD algorithm and not upset matrix of the layout of the image, so better effect. In the experiment data and attract our attention is $\mathrm{NC}$ value, from two can be seen in the graph, the experiment of NC value for were greater than 0.8. To sum up, from shearing Angle, based on SVD and DFT double watermarking scheme is feasible and robust.

\subsection{Rotation attack experiment.}

Figure 5 (a) said rotation Angle for 180 degrees to contain the watermark image, then the PSNR $=9.6404 \mathrm{~dB}$. Through the figure $5(\mathrm{~b})$ can see extracted watermark is 
very clear, the $\mathrm{NC}=0.9986$. Through the table 3 of the specific experimental data can be found that when rotation Angle for 270 degrees can also be clearly to extract the existence of watermark.

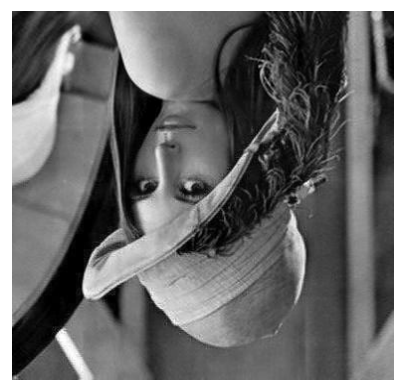

(a)

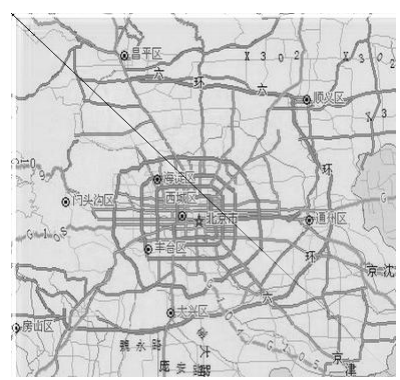

(b)

Figure 5 After rotate 270 degrees of the complex carrier images and watermarking extraction

TABLE III Contain the watermark grayscale image rotation of experimental data

\begin{tabular}{cc|c|c}
$\begin{array}{c}\text { Rotation } \\
\text { rate } \\
\text { content }\end{array}$ & $90^{\circ}$ & $180^{\circ}$ & $270^{\circ}$ \\
\hline PSNR & 26.1610 & 25.8524 & 26.1603 \\
\hline NC & 0.9987 & 0.9986 & 0.9987 \\
\hline
\end{tabular}

According to the above data, analysis, for rotating images, the position is changed, but the carrier image and the content does not change greatly, peak value signal-to-noise ratio of PSNR numerical value or high; For watermark for, due to its relatively fixed position, so even after rotating extracted watermark, and its normalization correlation coefficient is also very high $\mathrm{NC}$, namely the scheme has strong robustness.

\subsection{Noise against the experiment.}

Use imnoise () function in the already watermarking images adding noise density of 0.02 of salt \&pepper noise, the image more particles. Although the background image watermarking extraction with gray print, but still can see the extracted watermark figure 6 (b), then $\mathrm{NC}=0.9992$, PSNR $=18.5464$; Again the complex carrier images to join gaussian noise, set to 0 mean and variance in turn increased from 0.01 to 0.05 , the use of imnoise () function in the already watermarking images adding noise density of 0.02 of the gaussian noise, the image have greater particles. Although the background picture watermarking extraction 
with gray print, but still can see the extracted watermark figure 6 (d), then $\mathrm{NC}=$ 0.9993 , PSNR $=13.9862$; Through the table 4 experimental data can see, this algorithm has good anti-noise ability.

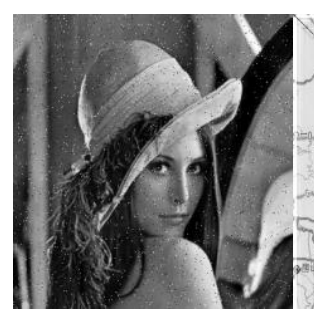

(a)

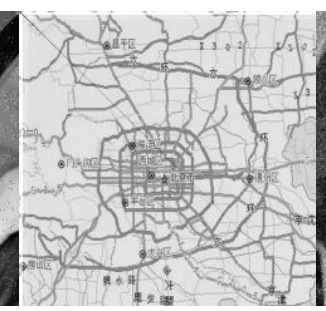

(b)

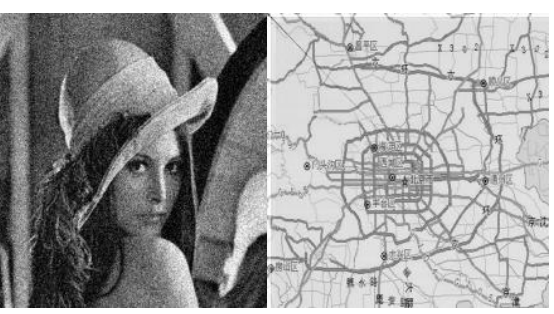

(c) (d)

Figure 6 Adding noise against the carrier image and complex after watermarking extraction ((a) superposition 0.02 salt \&pepper noise attack of the complex carrier images; (b) watermarking extraction; (c) watermarking extraction addingnoise density of 0.02 of the gaussian noise after the attack of the complex carrier images; (d) watermarking extraction)

TABLE IV Carrier watermark image noise experimental data

\begin{tabular}{c|c|c|c|c|c}
\hline $\begin{array}{r}\text { Noise or } \\
\text { cariance }\end{array}$ & 0.01 & 0.02 & 0.03 & 0.04 & 0.05 \\
\hline $\begin{array}{c}\text { PSNR } \\
\text { (Salt \&pepper) }\end{array}$ & 21.4525 & 18.5464 & 16.6014 & 15.5503 & 14.5999 \\
\hline $\begin{array}{c}\text { NC } \\
\text { (Salt \&pepper) }\end{array}$ & 0.9991 & 0.9992 & 0.9992 & 0.9993 & 0.9993 \\
\hline $\begin{array}{c}\text { PSNR } \\
\text { (Gaussian) }\end{array}$ & 13.9900 & 13.9862 & 14.0164 & 14.0209 & 13.9915 \\
\hline $\begin{array}{c}\text { NC } \\
\text { (Gaussian ) }\end{array}$ & 0.9992 & 0.9993 & 0.9992 & 0.9992 & 0.9992 \\
\hline
\end{tabular}

From the above data can see, whether salt \&pepper noise or gaussian noise, which are based on the original image and carrier images of the signal-to-noise ratio of the peak is not very big, this is mainly because the carrier image in the embedded after the noise, the image has changed, reflected in the visual Angle from will also be able to see, this just become we judge by the noise of the image is a basis for attack. And PSNR different is, whether salt \&pepper noise or gaussian noise, its normalization correlation coefficient $\mathrm{NC}$ value is very big still, watermarking extraction that with the original image is very close, this is what we expected.

\section{Summary}

This paper put forward based on the singular value decomposition and discrete Fourier transform with the combination of double watermarking algorithm, based on the carrier image watermark embedding and extracting double figures, and its 
feasibility was verified. In order to verify the algorithm is robust, the use of watermark image quality objective evaluation PSNR (peak signal to noise ratio) and $\mathrm{NC}$ (normalized correlation coefficient) to the algorithm simulation experiment, the experimental results show that this method can achieve good image visual effect, to shear, noise, and rotated against all has good robustness. The watermark algorithm can also used in digital audio and digital video and other digital media copyright protection for digital watermarking research opens up a new train of thought.

\section{References}

1. Peter H.W.Wong, Oscar C.Au, Y.M.Yeung.A Novel Blind Multiple Watermarking Technique for Images. IEEE Transactions on Circuits and Systems for Video Technology,VOL.13, NO.8, AUGUST 2003, pp.813-830.

2. S.Alexander, D.Scott, E.Ahmet. Robust DCT-SVD domain image watermarking for copyright protectio n:embedding data in all frequencies- [C]. 13th Euorpean signal processing conference. Antalya,Turkey: EUSIPCO2005, 2005.

3. C C Chang, Y Tsaip, C C LIN. SVD-based digital image watermarking- scheme[J]. PaRern recognition leRers, 2005,26:1577-1586.

4. X.Y.Xu, Y.Li. A digital watermarking algorithm based on the combination of DWT and SVD. Journal of southwest university for nationalities, natural science edition. 2007,33(5): :1032-1033.

5. LIU Fenlin, GAO Shanqing, GE Xin. A Scheme of 、 FragileWatermarking Based on SVD and 2D ChaoticMapping [J]. Journal of Shanghai Jiao tong University (Science), 2006,11(2):146-151.

6. Emir Ganic, Ahmet M.Eskicioglu. Robust DWT-SVD Domain Image Watermarking: Embedding Data in All Freque ncies. ACM,2004,9:166-174.

7. F J Huang,Z H Guan. A hybrid SVD-DCT watermarking method based on LP-SNR[J]. Pattern recognition letters, 2004, 25:1769-1775. 